\title{
Acute Myocarditis Mimicking ST- Elevation Myocardial Infarction in an 18-Year-Old Patient with Thyroiditis
}

\section{(1) Huseyin Ede1, (1) Shabir Ali Haider1, (1) Imad Awwad Ahmed2, (1) Haisam Hamdi Alsadi1}

${ }^{1}$ Hamad Medical Corporation Heart Hospital, Department of Cardiology, Doha, Qatar

${ }^{2}$ Hamad Medical Corporation Heart Hospital, Department of Radiology, Doha, Qatar

\begin{abstract}
Acute myocarditis can manifest in a different clinical form ranging from subclinical disease to death. In these clinical presentations, acute myocarditis can easily be misdiagnosed as acute ST-elevation myocardial infarction. Therefore, its differential diagnosis is very crucial in the management. Acute thyroiditis may also have similar pathobiological causes as acute myocarditis but the concomitant occurrence is very rare. Here, we presented a case of acute myocarditis mimicking ST-segment elevation myocardial infarction in an 18-year-old patient with concomitant acute thyroiditis and initial normal inflammatory markers.
\end{abstract}

Keywords: Acute myocardial infarction, myocarditis, thyroiditis, echocardiography

\section{Introduction}

Acute myocarditis develops due to inflammation of cardiac muscle and it needs differential diagnosis ${ }^{(1)}$. Its clinical presentation varies from subclinical disease to heart failure, cardiogenic shock, and even death ${ }^{(2)}$. The symptoms such as fatigue, dyspnea, peripheral edema, and chest discomfort usually develop over weeks and months. However, typical acute-onset angina can also be observed in patients with acute myocarditis although not commonly. Additionally, it can sometimes mimic

Address for Correspondence: Huseyin Ede, Hamad Medical Corporation Heart Hospital, Department of Cardiology, Doha, Qatar e-mail: huseyinede@gmail.com ORCID: orcid.org/0000-0003-1218-257X

Received: 08.09.2021 Accepted: 16.10.2021

Cite this article as: Ede H, Haider SA, Ahmed IA, Alsadi HH. Acute Myocarditis Mimicking ST-Elevation Myocardial Infarction in an 18-Year-Old Patient with Thyroiditis. EJCM 2021;9(4):207-212. 
acute coronary syndrome with ST elevation myocardial infarction (ACS/STEMI).

Thyroiditis is commonly associated with viral and bacterial pathogens, autoimmune diseases, drugs, radiation, or trauma ${ }^{(3)}$. However, acute myocarditis with concomitant acute-onset thyroiditis with normal inflammatory markers at presentation is very rare in the literature. Here, we presented an 18-year-old male patient with ACS/STEMI-like acute myocarditis and thyroiditis without obviously elevated inflammatory biomarkers or preceding respiratory tract infection.

\section{Case Report}

An 18-year-old male patient without any significant past medical history was brought to Emergency Department due to a new-onset, retrosternal, pressure-type chest pain lasting for 3 hours, with hotline electrocardiography (ECG) finding showing $0.5 \mathrm{~mm}$ ST depression over V1 and aVR and $2 \mathrm{~mm}$ ST elevation over II, III, aVF (Figure 1a). On arrival in the emergency department (ED), the patient was free of chest pain with similar ST changes as the hotline ECG (Figure 1b). Physical examination showed blood pressure of 107/57 $\mathrm{mmHg}$, heart rate of 111 bpm with oxygen saturation of $97 \%$ at room air. Chest and cardiac exams were unremarkable except for mild tachycardia.

Bedside point-of-care ultrasonography (POCUS) revealed normal left ventricular ejection fraction (LVEF) without regional wall motion abnormalities or pericardial effusion. An urgent detailed echocardiographic exam confirmed similar findings as those of POCUS (LVEF of $56 \%$ ). Initially, the patient was managed conservatively with differential diagnoses of perimyocarditis and acute coronary syndrome.

In the laboratory findings, the white blood cell count was $9.210^{3} / \mu \mathrm{L}$, hemoglobin was $13.4 \mathrm{gr} / \mathrm{dL}$, highsensitivity troponin $\mathrm{T}$ levels were significantly elevated (1312 ng/L at admission, $1155 \mathrm{ng} / \mathrm{L}$ after three hours, and $2828 \mathrm{ng} / \mathrm{L}$ after 24 hours after the admission with normal renal function). Alanine transaminase and aspartate transaminase levels were $32 \mathrm{U} / \mathrm{L}$ and $113 \mathrm{U} / \mathrm{L}$, respectively, with normal bilirubin level $(14 \mu \mathrm{mol} / \mathrm{L})$ (Table 1). The patient had total cholesterol level of $4.1 \mathrm{mmol} / \mathrm{L}$, LDL of $2.7 \mathrm{mmol} / \mathrm{L}$, triglyceride of $0.5 \mathrm{mmol} / \mathrm{L}$ and $\mathrm{HDL}$ of 1.2 $\mathrm{mmol} / \mathrm{L}$. Serum C-reactive protein (CRP) level was 2.6 $\mathrm{mg} / \mathrm{L}$, procalcitonin $0.02 \mathrm{ng} / \mathrm{mL}$ at admission.

The patient developed similar chest pain with more prominent ST elevation over II, III, aVF, V5, V6, V7, V9) (Figures 1c and d) 3 hours after the arrival in ED. The patient was promptly transferred to the cardiac catheter laboratory to rule out possible coronary artery-related acute coronary syndrome while he was having ongoing chest pain. The coronary angiogram showed normal epicardial coronary arteries without spasm or dissection (Figure 2). Acute myocarditis treatment was initiated with ibuprofen $400 \mathrm{mg}$ tablet three times a day and colchicine $0.5 \mathrm{mg}$ tablet twice a day along with metoprolol 12.5 $\mathrm{mg}$ twice a day. Cardiac magnetic resonance imaging (CMRI) was performed 48 hours after the admission. It showed high normal LV volumes with moderately impaired systolic function (LVEF 42\%), basal-to-apical anterior, lateral, and inferior walls hypokinesia with corresponding myocardial edema, subepicardial early and delayed hyperenhancement (Figure 3). The diagnosis was confirmed accordingly. Subsequently, ibuprofen was changed to aspirin $600 \mathrm{mg}$ tablet three times a day and lisinopril $2.5 \mathrm{mg}$ daily was added to the treatment plan. At follow-up, the results of respiratory panel, tested via polymerase chain reaction (PCR) (including influenza virus, parainfluenza virus, adenovirus, coronavirus disease-2019 (COVID-19), MERS coronavirus, human bocavirus, human enterovirus, human rhinovirus, the respiratory syncytial virus, Bordetella pertussis, legionella pneumophila, and human metapneumovirus), were unremarkable in nasopharyngeal swab. Additionally, the results of antineutrophil cytoplasmic antibody and antinuclear antibody blood tests were negative.

Coincidentally, the patient was diagnosed with painless, overt hyperthyroidism (thyroid-stimulating hormone (TSH) of $0.03 \mathrm{mIU} / \mathrm{L}, \mathrm{FT} 4$ of $35.2 \mathrm{pmol} / \mathrm{L}$ and 

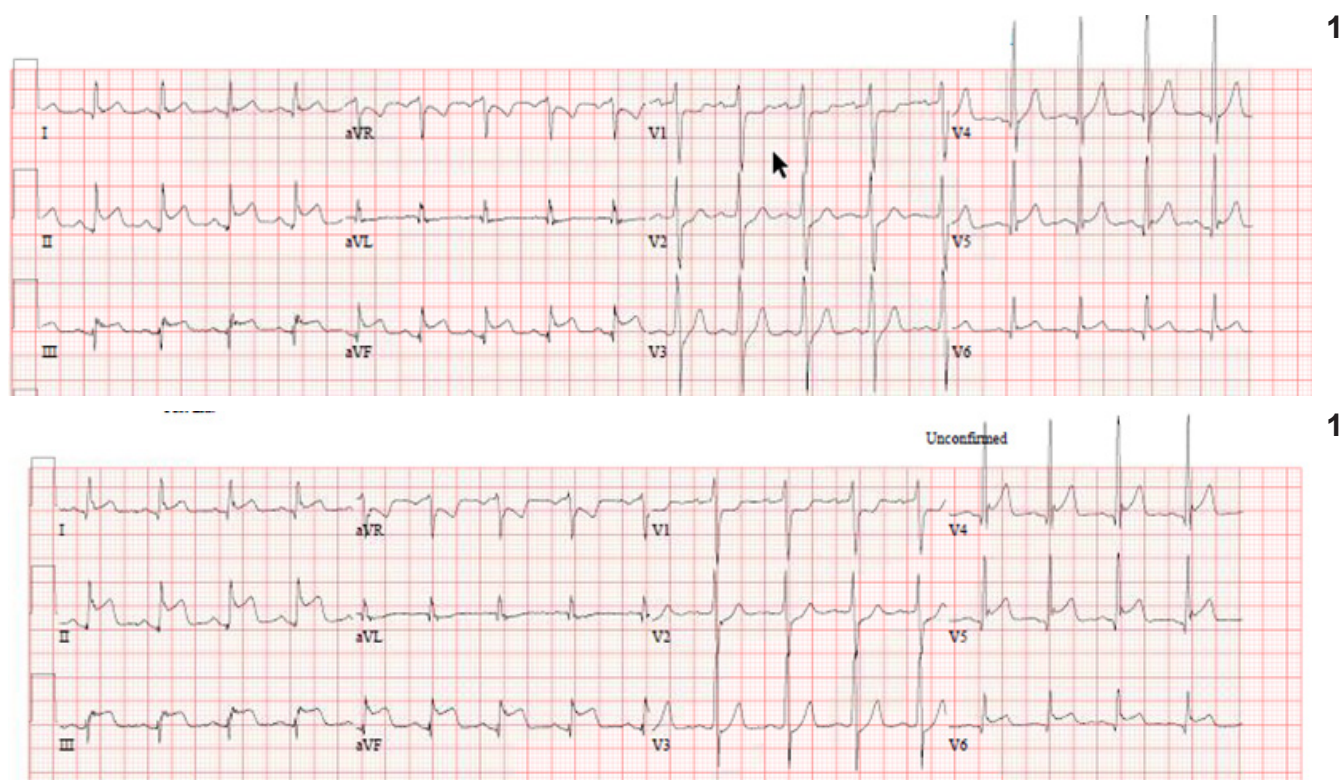

$1 b$

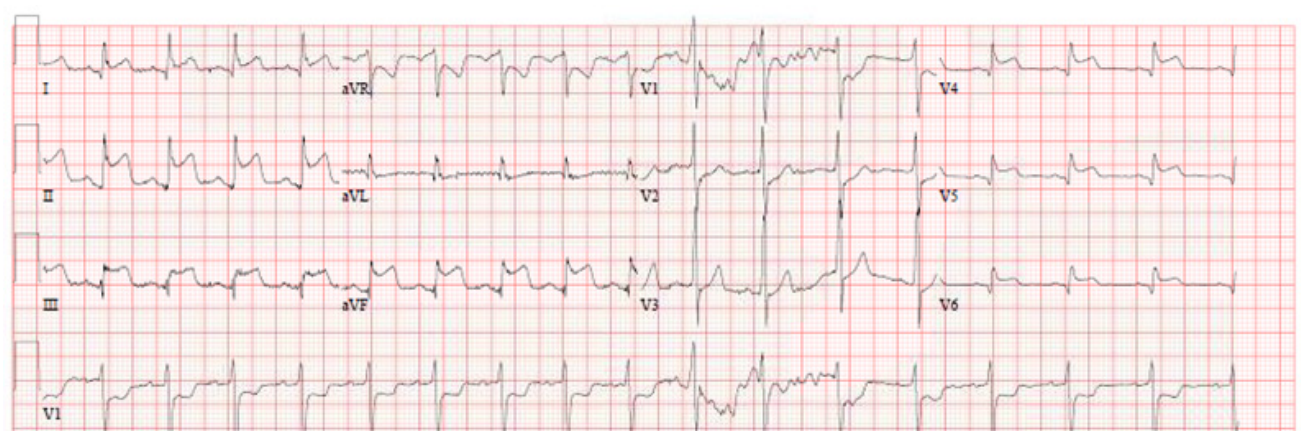

1c

$1 d$
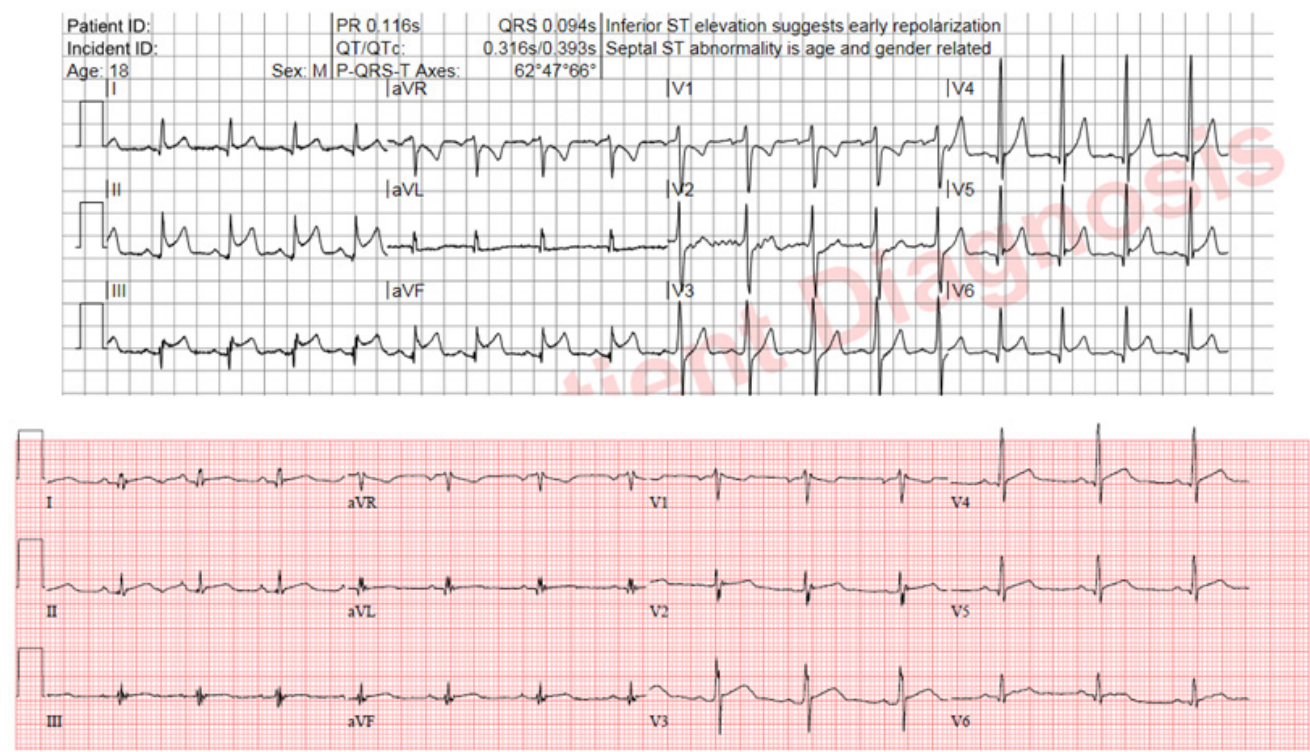

$1 e$

Figure 1a. Hotline electrocardiography (ECG) by the ambulance team; 1b) ECG at arrival; 1c) standard 12-lead ECG when chest pain developed 3 hours after the arrival; 1d) ECG with posterior leads; V4, V5, and V6 stand for V7, V8, and V9 respectively; 1e) 12-lead ECG at discharge in the $6^{\text {th }}$ day. 


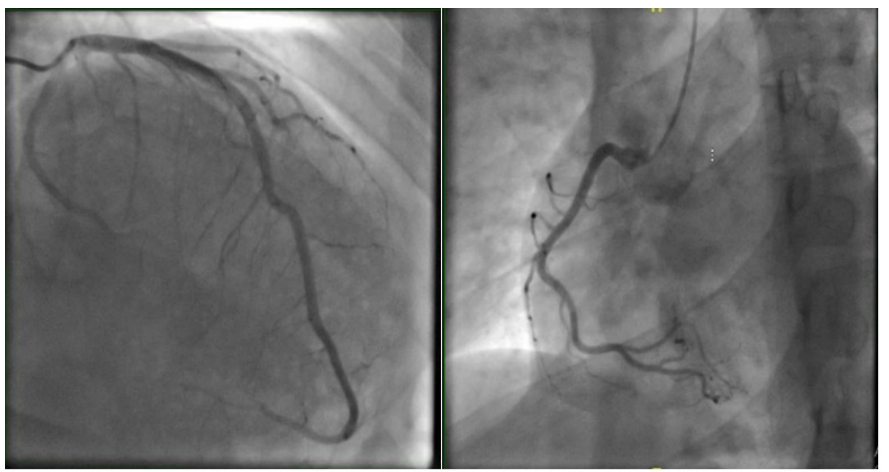

Figure 2. The coronary angiogram showed normal coronary artery
FT3 of $33.9 \mathrm{pmol} / \mathrm{L}$ at the admission) with the absence of anti-thyroid peroxidase or TSH receptor antibodies. The thyroid function tests improved within the following days without starting any anti-thyroid agent. (TSH of 0.04 $\mathrm{mIU} / \mathrm{L}, \mathrm{FT} 4$ of $13.3 \mathrm{pmol} / \mathrm{L}$ at day 5 after the admission) (Table 1). Both lobes of the thyroid gland and isthmus region were normal in size and shape with normal parenchymal echotexture and no focal lesions in thyroid ultrasonography. Additionally, few cervical lymph nodes were detected bilaterally, in the right side measuring around $12 \times 4 \mathrm{~mm}$ and in the left side measuring around

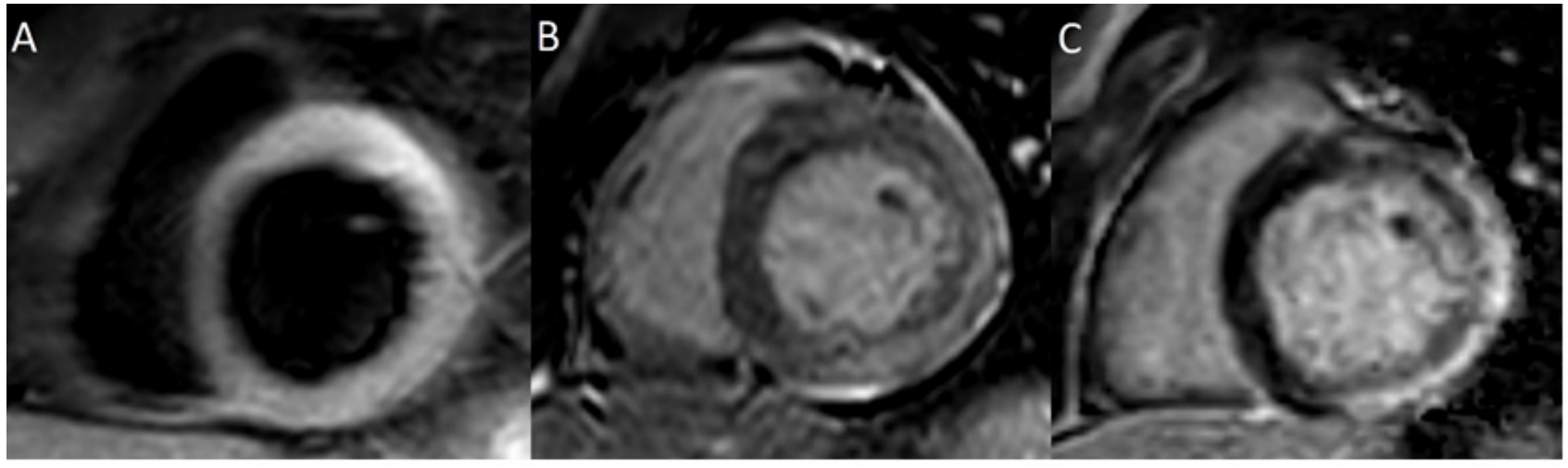

Figure 3. Cardiac magnetic resonance imaging showed the changes in consistent with acute myocarditis

Table 1. Laboratory and clinical findings of the patient during the follow-up in the hospital

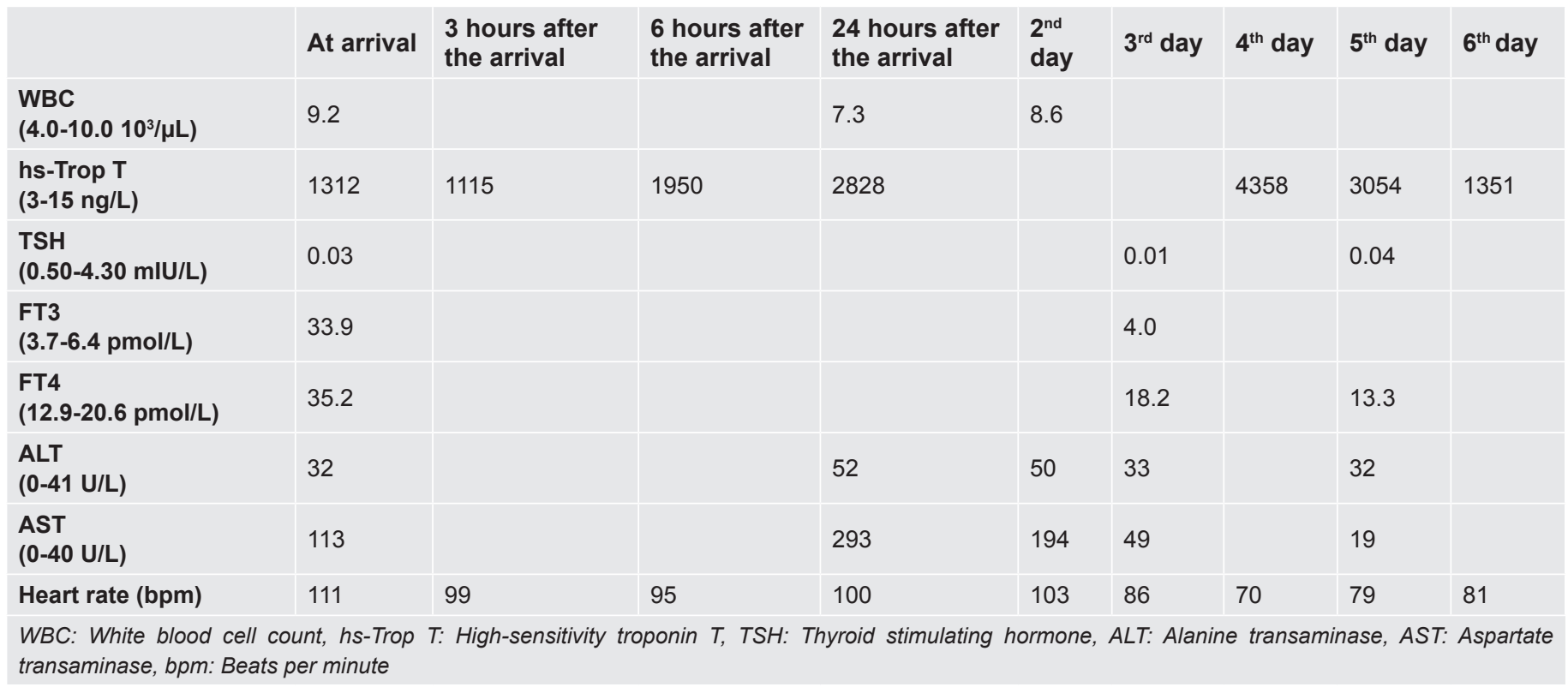


$16 \times 4 \mathrm{~mm}$ in size. Thus, the findings were consistent with acute thyroiditis.

The patient was discharged in stable condition with aspirin, colchicine, lisinopril $2.5 \mathrm{mg}$ daily, and metoprolol $25 \mathrm{mg}$ twice a day and with evolving ECG at day 6 (Figure $1 \mathrm{e})$.

\section{Discussion}

In this case report, we presented concomitant acute myocarditis and acute thyroiditis without obvious inflammatory status, mimicking acute coronary syndrome in an 18-year-old male patient. We aimed to share our experience in diagnosis, treatment, and follow-up of such a young patient.

Acute myocarditis can mimic acute coronary syndrome $^{(4)}$. As in this case, it is very easy to mix the diagnosis with acute myocardial infarction, considering his initial manifestations such as dynamic ECG changes with recurrent, typical, burning-like retrosternal angina, and normal CRP in absence of PR segment deviation, fever or recent upper respiratory infection.

Considering the initial ECG changes (ST-segment elevation without PR deviation), it can be clearly stated that the patient was at the beginning stage of acute myocarditis. As per the literature, transient ST elevation is often seen and disappears within 48 hours in $74 \%$ of all patients manifested with initial ST-segment elevation ${ }^{(5)}$. In our case, ST elevation at the admission almost disappeared on the day $6^{\text {th }}$.

It is very rare to develop ACS/STEMI at young age in the absence of risk factors. In general, less than $10 \%$ of patients with myocardial infarction are younger than 40 years old ${ }^{(6)}$. In the literature, ACS/STEMI at a young age without prominent risk factors (21 and 27 years old patients respectively) were reported previously ${ }^{(7)}$. Our case was 18 years old, without risk factors. with the recurrence of chest pain, coronary angiography (CAG) was performed and showed normal coronaries. This case was not an example of myocardial infarction (MI) with non-obstructive coronary arteries (MINOCA) since CMRI revealed changes that were consistent with myocarditis.
Another interesting finding of the patient was mild sinus tachycardia at the admission and at the following hours in the absence of any chest pain or heart failure symptoms although it is commonly observed in acute myocarditis, especially as a warning sign of heart failure and shock. Interestingly, the patient had concomitant overt hyperthyroidism due to acute thyroiditis along with acute myocarditis. Thyroid functions returned to near normal at day 5 without any intervention. Concomitant myocarditis and thyroiditis may develop in autoimmune diseases or drug exposure in animal models ${ }^{(7)}$; but are not described in humans. Autoimmune biomarkers were negative in our patient. Viral pathogens are the most common reason for acute myocarditis or thyroiditis ${ }^{(1,8)}$; but it is very difficult to isolate the causative agent most of the times. In our case, we also could not isolate any viral pathogen.

In conclusion, acute myocarditis and acute thyroiditis can be observed in the same patient. Thyroiditis-related hyperthyroidism should be suspected in an unexplained tachycardia at presentation. Myocarditis should be considered in the differential diagnosis of acute angina at a young age, especially in the absence of obvious risk factors regardless of initial inflammatory marker levels.

\section{Acknowledgements}

Ethical and institutional approvals were obtained (MRC-04-21-656, dated on 06/09/2021).

\section{Ethics}

Informed Consent: It was obtained from the patient.

Peer-review: Externally peer-reviewed.

\section{Authorship Contributions}

Surgical and Medical Practices: H.E., S.A.H., I.A.A., H.H.A., Concept: H.E., S.A.H., I.A.A., H.H.A., Design: H.E., S.A.H., I.A.A., H.H.A., Data Collection and/or Processing: H.E., I.A.A., Analysis and/or Interpretation: H.E., S.A.H., H.H.A., Literature Search: H.E., S.A.H., I.A.A., H.H.A., Writing: H.E., S.A.H., I.A.A., H.H.A.

Conflict of Interest: The authors declare that they have no conflict of interest. 
Financial Disclosure: The authors declare no financial support by any grant or research sponsor and no competing financial interest.

\section{References}

1. Caforio AL, Pankuweit S, Arbustini E, et al. Current state of knowledge on aetiology, diagnosis, management, and therapy of myocarditis: a position statement of the European Society of Cardiology Working Group on Myocardial and Pericardial Diseases. Eur Heart J 2013;34:2636-48.

2. Ammirati E, Frigerio M, Adler ED, et al. Management of acute myocarditis and chronic inflammatory cardiomyopathy: an expert consensus document. Circ Heart Fail 2020;13:e007405.

3. Pearce EN, Farwell AP, Braverman LE. Thyroiditis. N Engl J Med 2003;348:2646-55
4. Buttà C, Zappia L, Laterra G, Roberto M. Diagnostic and prognostic role of electrocardiogram in acute myocarditis: A comprehensive review. Ann Noninvasive Electrocardiol 2020;25:e12726.

5. Di Bella G, Florian A, Oreto L, e al. Electrocardiographic findings and myocardial damage in acute myocarditis detected by cardiac magnetic resonance. Clin Res Cardiol 2012;101:617-24.

6. Egred M, Viswanathan G, Davis GK. Myocardial infarction in young adults. Postgrad Med J 2005;81:741-5.

7. Prud'homme GJ, Parfrey NA, Vanier LE. Cyclosporine-induced autoimmunity and immune hyperreactivity. Autoimmunity 1991;9:345-56.

8. Kamar N, Marion O, Abravanel F, Izopet J, Dalton HR. Extrahepatic manifestations of hepatitis E virus. Liver Int 2016;36:467-72. 